\title{
Das Larvenstadium von Ascetta primordialis und Ascetta clathrus.
}

\author{
Von \\ Osear Sehmidt.
}

Mit Tafel XV und XVI.

1. Haeckel, Kalkschwämme. 1872.

2. Oscar Schmidt, Zur Orientirung über die Entwickelung der Spongien. Zeitschrift f. wissensch. Zoologie. XXV. Supplement. 1875.

3. F. E. Schulze, Ueber den Bau und die Entwickelung von Sycandra raphanus. Haeckel. Ebendaselbst.

4. Oscar Schmidt, Nochmals die Gastrula der Kalkschwämme. Archiv f. mikrosk. Anatomie. Bd. 12. 1876.

5. Barrois, Embryologie de quelques Éponges de la Manchc. Annales des Sciences nat. 6e Serie. P. 3. 1876.

6. Metschnikoff, Beiträge zur Morphologie der Spongien. Zeitschr. f. wissensch. Zoologie. Bd. 27. 1876.

7. F. E. Schulze, Zur Entwickelungsgeschichte v. Sycandra. Ebendaselbst.

8. Keller, Untersuchungen über die Anatomie u. Entwickelungsgeschichte einiger Spongien des Mittelmeeres. Basel 1876,

9. Haeckel, Studien zur Gasträa-Theorie. Jena 1877.

Meine Beobachtungen aber die Larven von Sycandra raphanus und glabra (2) liefen in dem Satze zusammen, dass beide Arten keine Gastrula bildeten. Ich bestätigte Metschnikoff's Angabe, dass die Larve sich mit dem geissellosen Hintertheile festsetze und sah wenigstens in einem Falle, dass nach dem Ansetzen die grossen körnigen Zellen von einer wechselnd dicken Schichte Protoplasma als einer Aussenschicht bedeckt waren. Ich irrte, als ich dieses Protoplasma aus den Körnerballen ausgetreten glaubte, indem ich 
durch Metschnik off's, jetzt sicher als unrichtig erkannte Meinung befangen war, wonach die Geisselzellen sich zum Entoderm einsttilpen sollten.

Unmittelbar darauf wurde durch F. E. Schulze (3) die Gastrula von Sycandra raphanus wieder zu Ehren gebracht, bis, auch nur ein Jahr später, Barroi s (5) erklürte, die Gastrula folge nicht auf das bekannte Larvenstadium, wie Schulze dargestellt, sondern gehe der amphiblastula-förmigen Stufe voraus, sei ein vorübergehender Zustand und für die definitive Entwickelung und die Organanlage der betreffenden Kalkschwämme nur von untergeordneter oder keiner Bedeutung. Auch Schulze beeilte sich zu erklären (7), er habe sich bei erneutem Studium überzeugt, dass, wie es Barro is angebe, aus der Amphiblastulaform zunächst durch Einsttilpung des dunkelkörnigen Zellenlagers sogleich die Gastrula, und aus dieser dann erst durch gewaltiges Auswachsen und Wiedernachaussendringen der dunkelkörmigen Zellen die oft beschriebene freie eiförmige Larve entsteht.

Beide Beobachter sind also bei dem Punkte angelangt, von wo aus ich den definitiven Schwamm hervorgehen liess, und ich möchte nur Barrois' Angabe, dass meine kurze Vertheidigung (4) „ne contient pas d'observations nouvelles", dahin berichtigen, dass ich darin doch die für die ganze Frage nicht unwichtige Beobachtung veröffentlichte: dass die jüngsten festsitzenden Ascandra coriacea und variabilis mundlos sind. Diese Thatsache ist von ihm selbst auch an anderen Kalkschwämmen bestätigt und nach ihrer Bedeutung gewtirdigt.

Gleichzeitig hatte Keller (8) seine Untersuchungen angestellt. Sie waren vor der Publication der Barrois'schen abgeschlossen. Er macht die Geisselhälfte der Larve zum Hinterende, lässt aus der Larve eine Gastrula entstehen und dieselbe sich in der Weise festsetzen, wie Haeckel es sich vorgestellt hatte, jedoch mit Verschluss des primitiven Mundes. Er hält, auch nach Bekanntwerden von Barrois' Beobachtungen an dem Uebergang der Gastrula in den fertigen Zustand fest, befindet sich aber hinsichtlich dieses Punktes jetzt auch mit Schulze, hinsichtlich der Ansatzstelle mit allen anderen Beobachtern, Karter einbegriffen, im Widerspruch. Schulze ist unentschieden.

Die Bildung einer echten Gastrula, wie Kell er solche annimmt, halte ich noch heute fur eben so unrichtig, wie ich es da- 
mals Schulze gegentiber gethan. Bei der Veröffentlichung seiner Arbeit konnte sich Keller hinsichtlich des Ueberganges der Larve in den jungen Schwamm noch auf Schulze berufen. Seitdem der letztere sioh mit Barrois nach erneuten Beobachtungen einverstanden erklärt, steht Kell er allein. Auch widerspricht Haeckels neueste Aeusserung (7), dass durch die meisten andern Beobachter die Existenz der Gastrula bei den Kalkschwämmen festgestellt sei, dem literarischen Thatbestande. Denn selbst wenn man das voribergehende Einstillpungsstadium nach Barro is und Sch ulze gelten lassen will, so wäre dasselbe doch keine Gastrula nach dem von Ha eckel selbst umgrenzten Begriffe. Eine sack- oder schtisselförmige Larve, deren Vertiefung für die spätere Entwicklung ohne die geringste Bedeutung ist, weder mit den Darmöffnungen noch mit irgend einem Theile des Darm- und Canalsystems die geringste Beziehung hat oder irgend einen Einfluss ausibt, ist keine Gastrula. Zu diesem Resultat ist Barro is gelangt. „Les cavités de l'éponge adulte n'ont pas plus le rapport avec la cavité de segmentation qu'avee la cavité d'invagination de la larve; l'oscule de l'adulte, qui ne se formera que plus tard, n'a pas non plus de rapport avec la bouche de la larve; il n'y a done aucune homologie entre l'oscule ou le cloaque des Éponges et la bouche des Zoophytes." Dieser Vergleich und, diese Homologie war die Hauptsache, auf welche es Haeckel ankam. Mit Ausnahme von Keller sind wir aber jetzt Alle der Ueberzeugung, ich seit meiner Angabe in 1 uber Kalk- und Kieselschwämme, dass diese Homologie, welche den Uebergang der Gastrula in die definitive Form voraussetzt, nicht existirt. Allerdings kommen Diejenigen, welche an der Homologie des Canalsystems der Schwämme mit dem der Gastrovascularapparat der Coelenteraten festhalten, die Beobachtungen Kow alewsky's in seiner russischen Abhandlung von 1873 uber Campanularia zu Statten, wo, soweit sich aus den Bildern urtheilen lässt, die Höhlungen der Campanularie sich im Entoderm ähnlich zeigen, wie die Wimperkörbe und Wimpercanäle bei den jungen Spongien. Auch sind Kowalewsky's Untersuchungen iber die Brachiopoden von 1874, so weit es sich aus den Abbildungen entnehmen lässt, hierher zu ziehen. Was bei Argiope durch eine Invagination der weitschichtigen embryonalen Blase zu Stande kommt, die Beschaffung des von vorn herein häutigen Entoderms als des Materials ftir Darm und Leibeshöhle, geschieht bei Theci- 
dium durch Bildung eines Zellenhaufens vermittelst Wanderzellen aus dem Exoderm. Wir könnten uns also nicht wundern, wenn bei einigen Spongien die ächte Invaginations-Gastrula entdeckt wurde, obschon bei der Mehrzahl und in allen bis jetzt mit einiger Sicherheit beobachteten Fällen die Entwickelung anders verläuft.

Meine Beobachtungen uber Ascetta clathrus standen bisher nicht nur mit Haeckels Mittheilungen ther diese Art und uber Asculmis armata in Widerspruch, sondern schienen iberhaupt isolirt und mit dem anderweitig Erforschten nicht vereinbar zu sein, namentlich auch, seitdem Barro is' Abbildung der Flimmerlarve von Ascandra contorta (כ. Fig. 2) sich eng an die der ubrigen von ihm untersuchten Kalkschwämme, sowohl Leuconen als Syconen anschliesst. Ich habe nun während eines abermaligen Aufenthaltes in der zoologischen Station in Neapel von Februar bis Mai 1877 nicht nur die Untersuchung von Ascetta clathrus revidiren, sondern auch auf Ascetta primordialis ausdehnen können. Es liess sich nicht zu einem Abschluss gelangen, allein die höchst wichtige Periode der Bildung des Entoderms ${ }^{1}$ ) glaube ich nunmehr an beiden Arten klar darstellen zu können, und es wird sich daraus ein Schluss auf die ontogenetische Stellung der amphiblastulaförmigen Larven der anderen Kalkspongien ziehen lassen.

Sehr vortheilhaft erwies sich auch diesmal eine kurze, nach Secunden, höchstens einigen Minuten zählende Behandlung der Flimmerlarve mit stark verdtinnter Ueberosmiumsäure. Hat man es gut getroffen, so brechen die Larven bei vorsichtiger Verschiebung des Deckglases so auseinander, dass man in die Innenfläche der einen Hälfte und a uf die daneben liegende andere Hälfte sieht. Unter meinen sparsam ausgewählten Abbildungen befinden sich daher keine optischen oder sonst combinirten Durchschnitte, sondern sie sind der gerade Ausdruck der sich direct darbietenden Flächen des Präparates.

Ich fubre zuerst die Larve von Ascetta primordialis vor (Fig. 1 bis 8). Meine Beobachtungen betreffen nicht die Furchung und die Bildung der Flimmerlarve, sondern beginnen mit dem Stadium,

1) Ich habe früher die Bezeichnung Entoderm für einen unregelmässigen Zellenhaufen vermieden und gesagt, ein Entoderm sei nicht da. Es wird jetzt an der Sache nichts geändert, indem ich das Wort in der von den meisten Histologen gebrauchten weiteren Bedeutung nehme. 
wo die Larve in Gestalt eines etwas platt gedrickten Ovals aus einer Schichte geisseltragender Cylinderzellen besteht. Diese Wand umgibt einen mit einer eiweissartigen Flïssigkeit erfüllten Raum, also die grosse primitive Furchungshöhle. Das Vorderende ist der stumpfere Eipol, und man zählt auf den grossen Durchmesser der dem blossen Auge als weisse Piunktehen sichtbaren Larven einige vierzig bis funfzig Geisselzellen von der bekannten Form und Beschaffenheit. Das den Kern enthaltende Vordertheil der Zelle hat einen körnchenlosen klaren Inhalt, die untere Hälfte ist mit den Pigmentkörperehen versehen. Nur in der Form und Länge weichen die Geisselzellen des Vordertheiles um ein Geringes von denen des Hintertheiles ab, indem die ersteren schlanker sind (Fig. 8). Die Larve ist also jetzt eine einschichtige Blase. Die erste Andeutung einer ktinftigen Veränderung besteht in einer gewissen Auflockerung der Zellen des Hinterpoles, wobei der Breitedurchmesser der betreffenden Zellen sich etwas vergrössert und die einzelnen Zellen sich mit ihren Kuppen mehr hervorwölben. Eine dieser Zellen aus der Nachbarschaft des Poles, nie die Polzelle selbst, wie man sie mathematisch bestimmen würde, eilt der andern voraus, erweitert sich unter Einziehung der Geissel und Verkürzung des Längsdurchmessers auf das Dreifache und bekommt einen grobkörnigen Inhalt, eine Verwandlung, welche unmittelbar darauf noch zwei andere neben ihr auf einer Seite, nicht rechts und links liegende Zellen durchzumachen pflegen (Fig. 1. 2). Da die Geisselzellen der Natur der Sache nach gleich den Steinen eines Gewölbes sich verschmälern, so werden die in der Umwandlung begriffenen und sich vergrössernden Zellen nothwendig etwas hervorgedrängt. Beim leisesten Druck, wodurch man den Inhalt der Larve gegen die Wand presst oder die Zellen gegen einander, erhält man solche Zustände und Bilder wie Fig. 3.

Im normalen Verlauf aber tritt jene vergrösserte Zelle durch eine mir nicht bekannte mechanische Nöthigung, vielleicht in Folge der Aufsaugung von Flüssigkeit aus der Furchungshöhle, nach innen, ein Moment, wie ich ihn gliicklicher Weise abgefasst habe (Fig. 4). Die Liicke, aus welcher die e rst e Ent od er mz ell e eingetreten, schliesst sich alsbald wieder und der Umwandlungsprocess der Ectodermzellen za einwandernden Entodermelementen hat seinenFortgang. Ein sehr instructives und überzeugendes Präparat ist in Fig. 5 wiedergegeben. Die Larve ist im Längsdurchmesser ausein- 
andergebrochen; man sieht in dem aus den Geisselzellen gebildeten Napfe die sechs bisher eingeschlïpften Entodermzellen. Bei reichlich mir zufliessendem Material habe ich alle möglichen Grade der Ausfüllung mit solehen Entodermzellen angetroffen. Wie das Präparat (Fig. 6) zeigt, die Ansicht einer Larvenhälfte von innen, sind diese Zellen nicht gleich gross, ein Unterschied, der schon bei der Anlage der ersten Wanderzellen hervortritt. Auch finden sie sich im ganzen Binnenraum zerstreut, nachdem eine grössere Anzahl eingewandert. Ich muss es unentschieden lassen, ob die Umwandlung und Einwanderung bloss von der einen Stelle des Hinterendes der Larve aus geschieht. Doch ist dies wahrscheinlich, da ich nie an einer anderen Stelle der Larve Spuren davon angetroffen. Eine Anordnung der eingewanderten Zellen zu einem regelmässigen Lager, einem eigentlichen Entoderm nach dem Sinne des Wortes, findet nicht statt.

Ich habe mir viele Mühe gegeben, theils durch öftere gänzliche Erneuerung des Wassers, theils durch continuirlichen langsamen Wasserwechsel die Larven in den definitiven Schwamm umzuwandeln, habe aber nur sehr unvollständige Resultate erzielt. Nach zwei bis drei Tagen wurden die Larven sesshaft in Form von flachen unregelmässigen Fladen (Fig. 7). Die Zellen des Ectoderms verkürzen sich unter Einziehung der Geissel und fangen an zu versehmelzen. Das wie eine Zwischenmasse erscheinende nunmehr gemeinschaftliche Protoplasma zieht sich in feine Fortsätze aus, die auch zum Theil direct aus den Geisseln hervorgegangen sind. Denn ich beobachtete wiederholt, dass die von ihrer Unterlage durch die mikroskopischen Hantierungen losgelösten Pseudopodien wieder die Form der Geissel annahmen. Letztere also hatten sich vorher theilweise zurtickgezogen, verdickt und dienten so zur Fixirung des Körpers. An der Mehrzahl der Exemplare konnte man auf diese Weise eine langsame amöbenartige Veränderung des Randes wahrnehmen. Ich beobachtete in einem Falle, dass der Rand sich im Verlaufe einer halben Stunde abgerundet hatte und der ganze Körper uhrglasförmig geworden war; alle Protoplasmafortsätze waren eingezogen, und die schon vermischten Zellenconturen wieder deutlich geworden. Es scheint jedoch, als ob schon auf diesem Stadium die Zahl der zum Syncytium sich verbindenden Zellen verringert sei. Dies war ganz entschieden bei dem Exemplare der Fall, von dem ich ein Stutck 
in Fig. 8 abgebildet. Zelle für Zelle ist gezeichnet, und da an eine Verwechslung mit einem andern Organismus nicht wohl zu denken, so bleibt nichts iibrig, als eine Verwachsung mehrerer Geisselzellen zu diesen grossen amöboiden Aussenschichtzellen anzunehmen. Ich werde unten darauf zurlickkommen, dass ich durchaus nicht der Ansicht bin, diese Zellen gingen schliesslich in die Pflasterzellen tiber, welche M. Schultze bei Sycandra raphanus beschreibt.

Allem Anschein nach gehört auch die auf der Unterlage aufliegende Partie der sich umwandelnden Larve dem Ectoderm an. Ueber das Schicksal des Entoderms weiss ich nichts zu sagen, als dass es als ein dunklerer Inhalt durch die Aussenschicht durehscheint. Würde die Verschmelzung des Syncytiums weiter vor sich gehen, so wäre die Uebereinstimmung mit dem jüngsten $\mathrm{Zu}$ stande der umgewandelten Sycandra compressa bei Barrois (4. Fig. 15) hergestellt. Das Auftreten der Nadeln habe ich jedoch weder bei Ascetta primordialis noch bei Ascetta clathrus beobachtet.

Ich wende mich nun zu dieser zweiten Art, welche wegen der Kleinheit der Ectodermzellen und des dieselben begleitenden grünen Pigmentes der Untersuchung einige Hindernisse bereitet. Vor zwei Jahren (2) habe ich festgestellt, dass die Abbildung Haeckels (1. Taf. 4. Fig. 6) von der ganzen Larve, abgesehen von der etwas zu gross gerathenen Länge der Geisseln richtig ist, dass aber der optische Längsdurchschnitt (Fig. 7) mit der schönen Entodermschichte auf Täuschung beruht, und dass man bei den ausgebildeten schwärmenden Larven statt dieser Schicht nur einen unregelmässigen Zellenhaufen findet. Diesen Angaben ist man mit entschiedenem Misstrauen begegnet, vorzigglich desshalb, weil eine Vergleichung mit den andern bekannten Kalkschwammlarven ausgeschlossen schien. Dass auf der andern Seite gerade eine Anknlipfung für die Larven der Kieselspongien und Halisarken gegeben war, blieb unbeachtet. Ich habe das Vergntigen, die vollkommene Uebereinstimmung der Larve der Ascetta clathrus mit derjenigen von Ascetta primordialis zu constatiren und meine damaligen Beobachtungen mit einigen unwesentlichen Modificationen aufrecht zu erhalten. Die erste Correctur meiner früheren Mittheilungen betrifft das griune Pigment. Dasselbe liegt nicht nur, wie ich glaubte, oberflächlich, gleichsam 
wie eine Ausscheidung auf den membranlosen, doch isolirbaren Cylinderzellen, sondern ist in Gestalt feiner contourirter Körnchen iiber nnd durch die ganze Zelle verbreitet, ja vorzugsweise im unteren Theile hinter dem Kerne angehäuft. Es fällt also meine Behauptung, dass eine, jedenfalls eng mit dem Ectoderm verbundene Schichte grïner Pigmentkörnchen von dem eigentlichen Ectoderm als eine Art Cuticularschicht unterschieden werden könne; und damit ist die Harmonie mit Ascetta primordialis völlig hergestellt. Man sieht in Fig. 12 und 14, wie die Körnchen in und auf der äusseren Grenzschicht des Zellenleibes sich befinden.

Wie oben erwähnt, ist es mir diesmal sehr gut gelungen, die Flimmerlarven zu spalten und zu sprengen und an Hälften oder kleineren Stücken der Wandung mich von ihrer Einschichtigkeit zu uberzeugen. Fig. 11 ist ein solches Stlick, aber schon mit einer Entoderm-Wanderzelle. Was ich vorher von der Entstehung und dem Orte der Entstehung der Entodermelemente gesagt habe, misste ich für diese Art wiederholen. Fig. 10 zeigt das Hinterende einer leise gedrickten aber unversehrten Larve, bei welcher zwei zum Einwandern fertige Zellen hervorgetreten sind. Bei Fig. 9 ist unter stärkerem Drucke das Hinterende geplatzt und ein ganzer Kranz soleher Zellen zum Vorschein gekommen. Ich bitte, diese Abbildung umzudrehen und neben $\mathrm{Haeck}$ els Flimmerlarve von Asculmis armata (1. Taf. 13. 5) zu halten, so wird man sich der Vermuthung nicht erwehren können, dass eine Verwechslung einer solchen gesprengten Larve mit einem normalen Gastrulazustande stattgefunden hat. Ich habe meine Darstellung von 1875 also nur dahin zu vervollständigen, dass der Zellenhaufen nicht wechselnd in dem einen oder dem anderen Ende des Hohlraums, sondern im Hinterende angetroffen wird. Die Zellen sind jedoch gleich nach der Einwanderung ausgezeichnet amöboid beweglich, so dass binnen 20 bis 30 Secunden Formen wie Fig. 13 in einander tibergehen. Es ist also nicht zu verwundern, wenn sich nicht selten einzelne dieser Zellen weiter von ihrer Ursprungsstelle entfernen, und man sie da und dort in der grossen Höhlung antrifft, wie die eine in Fig. 11. Es kommen auch Fälle vor, dass beim Zerzupfen eines Präparates sich einige Geisselzellen in Gesellschaft von einigen Entodermzellen so isoliren, wie in Fig. 14. Es würde aber nach Allem, was vorausgegangen, ganz verkehrt sein, wollte man sich daraus eine Entodermschicht oder gar eine Gastrula construiren. 
Es handelt sich nun darum, nach einem einheitlichen Plane oder einem Zusammenhange der so verschieden dargestellten und ohne Zweifel wirklich sehr verschieden sich verhaltenden Larven der Kalkschwämme zu suchen, wobei ich gegen Keller für bewiesen ansehen dari, dass die von Metsehnikoff, mir, Schulze und Barrois beschriebenen Larven sich nicht in Gastrula-Form, sondern in der bekannten Amphiblastula-Form und zwar mit dem Körnerzellenende festsetzen. Wir sind zunächst zur Vergleichung unserer beiden Asconen mit der von Barrois beobachteten Larve von Ascandra contorta (5. Fig. 21) eingeladen. Diese Vergleichung kann aber nur unter der Voraussetzung stattfinden, dass das Stadium, von welchem ich aus Mangel an Beobachtungen über den Verlauf der Furchung und überhaupt der friiheren Bildungen auszugehen gezwungen bin, das der einschichtigen Flimmerlarve, nicht etwa einem Amphiblastula-Stadium gefolgt ist, sondern in der Reihenfolge der Entwickelung von Ascetta primordialis und Ascetta clathrus der amphiblastula-förmigen Larve von Sycandra raphanus u. a. entspricht, dass sie also in gleicher Weise wie bei dieser das Endresultat der Furchung ist. Ich erlaube mir dabei, auf das von Barrois und Schulze behauptete Intermezzo der gastrulaartigen Invagination keine Rücksicht zu nehmen, da demselben selbst von diesen Forschern jede tiefere Bedeutung abgesprochen wird. Meine Voraussetzung ist aber deshalb kaum angreifbar, weil bei Halisarca und verschiedenen Horn- und Kieselschwämmen die directe Bildung der einschichtigen Flimmerlarve aus der Furchung vollständig vorliegt, und weil das bei unseren Asconen nunmehr folgende Stadium nun wirklich die besten Ankntipfungspunkte zur bisher vergeblich gesuchten Erklärung der caenogenetischen AmphiblastulaForm zu geben scheint. Wenn ich mich einmal gegen den Missbrauch und das allzuergiebige Berufen auf die von $\mathrm{Haeckel}$ ins Licht gestellte Caenogenese ausgesprochen, so will ich mich nattirlich damit nicht des Rechtes auf diese Betrachtungsweise tiberhaupt begeben haben und halte das damit verbundene Princip der verkürzten Entwickelung nach wie vor für eines der fruchtbarsten.

Man kann darüber streiten, ob Ascetta mit Dreistrahlern oder Ascyssa mit Stabnadeln die einfachere oder ursprïnglichere Form sei; jedenfalls sind beide sehr einfach; und bei der fabelhaften Formenwandelbarkeit einiger oder aller sogenannten Species von 
Ascetta, sowie der verhältnissmässigen Seltenheit und Einförmigkeit der nur in zwei Arten auseinandergehenden Ascyssa ist es nicht unwahrscheinlich, dass Ascyssa eine reducirte, verk uimmernde Ascortis, Asculmis oder Ascandra sei 1). Auch thut es der Einheit des Kalkschwammstammes keinen grossen Eintrag, wenn man sich die Stabnadeln von Ascyssa unabhängig entstanden denkt. In der Abtheilung der Kieselschwämme kann man der Annahme solcher Convergenzen kaum aus dem Wege gehen. Auf das jüngere Alter von Ascetta aus der grösseren Variabilität zu schliessen, wie wir sonst unter ähnlichen Verhältnissen bei Thier- und Pflanzenformen berechtigt sind, dürfte bei der allgemeinen Unstetigkeit der Schwammformen nicht erlaubt sein. Die Hexactinelliden der Gegenwart, wenn auch in der Abnahme, ja im Schwinden begriffen, variiren noch eben so, wie in der Kreide. Also ist gegen das hohe Alter von Ascetta-Arten nichts einzuwenden. Jedenfalls auch ist Haeckel im Recht, wenn er die Leuconen und Syconen von den Asconen ableitet. Niemand endlich wird der Annahme widersprechen, dass innerhalb der Asconen Ascandra eine jüngere und höhere Stellung einnimmt, als Ascetta. Im Gange von Barrois' Untersuchungen lag es, dass er die Larve seiner Ascandra contorta, welcher sich die von Keller behandelte Ascandra Lieberktihnii anreiht, mit der ihm wohlbekannten Larve von Sycandra verglich und ihre Homologien hervorzuheben suchte, und ich finde es begreiflieh, wenn er betreffs der Larve von Ascetta clathrus sagt: 0. Schmidt, qui a étudié récemment la même Ascetta clathrus, donne de ces embryons une description qui en rende difficile la comparaison avec les embryons des autres Éponges. Hatte ich doch selbst diese Vergleichung abgewiesen. Allein jetzt steht die Sache anders; die Larven der Asconen können und müssen zuerst unter einander verglichen werden. Es heisst von den Larven von Ascandra contorta: Les larves libres de Ascandra contorta, telles qu'on les voit lorsqu'elles nayent dans un aquarium où on a mis des éponges adultes dont les produits génitaux étaient mûrs, ressemblent entièrement à celles des Sycons précédemment décrits. - En dilacérant des Ascandra contorta adultes, j'ai pu observer des embryons plus jeunes; il est difficile de se procurer ainsi les oeufs en bon état, mais j'ai pu parfaitement reconnaître le stade qui suit la gastrula

1) So auch Haeckel. 
et précéde la larve libre, celui où les grosses cellules endodermiques quittent la cavité de segmentation. La figure 21 représente un de ces embryons, la cavité d'invagination a disparu, la cavité de segmentation est encore très spacieuse. Barrois erklärt also, dass er die Stufe nach der Gastrula gesehen und nimmt als selbstverständlich an, dass ein Gastrula-Stadium vorausgegangen sei, weil es so bei Sycandra war.

Setzen wir den Fall, der Beobachter wïsste noch nichts von der Entwickelung und den Larven der Leuconen und Syconen, ihm wären bisher nur meine beiden Asconen bekannt gewesen, und er entdeckte die so abweichende Form von Ascetta contorta: was würde er schliessen und schliessen müssen? Er würde die Gastrula nicht mit den Haaren herbeiziehen, auf die zu verfallen nicht die geringste Veranlassung vorliegt, sondern die einzige Möglichkeit eines Zusammenhanges von Ascetta und Ascandra darin finden, dass bei den Vorfahren von Ascandra contorta die ehemals secundäre Entodermbildung in verkürzter auf die Furchung ubertragener Entwickelung zu einer primären geworden ist. Barro is sagt, wir wiederholen: j'ai pu parfaitement reconnaître le stade qui suit la gastrula. Er hat sie aber nicht gesehen, diese imaginäre, im besten Falle unnütze Gastrula, die er hier voraussetzt, weil er sie bei Ascandra gesehen zu haben glaubt. Wer nun nach diesen Erfahrungen auf die Syconen wberginge, dem könnte es doch kaum einfallen, die schtisselförmigen und nach meiner Ueberzeugung rein zufälligen Vertiefungen durchaus als Gastrula zu retten. Es fehlt hierfür jede Nöthigung, jeder Vorwand, und ich hoffe, dass wir endlich diesen wesenlosen Schemen aus der Entwickelungsgeschichte der Spongien ausgemerzt haben. Alle Beobachtungen iber Halisarcinen und Kieselschwämme stimmen dazu.

Es scheint, dass $\mathrm{Haeckel}$ bei Abfassung des zweiten, 1877 veröffentlichten Theiles seiner Studien zur Gasträa-Theorie die Arbeit von Barrois und den bedeutsamen Widerruf Schulze's mit der Zustimmung zu Barrois Resultat noch nicht kannte. Er hätte sonst unmöglich die nur noch von Keller unterstutzte Behauptung nochmals vorbringen können (9. S. 260 ff.), der Urmund der Gastrula gehe bei den Spongien in den bleibenden Mund (osculum), der Urdarm, die Gastrulainvagination in den bleibenden Darm, d. h. das Canalsystem uber. Auch hätte er unmöglich die 
Gasträaden, vorausgesetzt, dass sie sich wirklich so entwickeln, wie er es dargestellt, also vorausgesetzt, dass ihm nicht eine ähnliche Täuschung, wie bei den Kalkschwämmen untergelaufen ist, unmöglich, sage ich, hätte er auf diese Entwicklung hin die Gasträaden für die allernächsten Verwandten der Spongien, gleichsam futr Spongien ohne Poren erklären dürfen. Von den von Haeckel beschriebenen Haliphysemen muss die eine ausgeschieden werden, Haliphysema echinoides. Haeckel nimmt nämlich irrthtimlich an, die Spicula seiner vier Exemplare seien aus verschiedenen Spongien zusammengewurfelt und als fremde Körper von Haliphysema zusammengeleimt. Dem ist nicht so. Ich habe schon in den „Spongien von Algier" zwei Stelletten, St. geodina und intermedia beschrieben, welche dieselben oder nahe die gleichen Kieselkörper wie die von dem vermeintlichen Haliphysema echinoides genuin enthalten, und in den "Spongien des atlantischen Oceans" sind weitere Beweise fir die Combination aller dieser Kieselformen bei Stelletten und Geodien enthalten. Die Stellung derselben in und an dem Körper des Haliphysema echinoides ist die naturgemässe. Wenn nun Haeckel dazu fügt, dass seine durch Koren empfangenen, von dem Tiefgrunde des atlantischen Meeres stammenden Exemplare auf der Basis einer Stelletta gesessen hätten, so wird man genöthigt sein, die Dinger erstens nicht für Haliphysemen, sondern fur Spongien, und zweitens mit grösster Wahrscheinlichkeit für Junge eben jener Stellette zu halten, auf der sie sich angesiedelt hatten oder auch vielleicht gesprosst waren. Eine Untersuchung jener Stelletta im Museum von Bergen kann dartiber entscheiden. Auf den Umstand, dass keine Poren gefunden wurden, ist, wie Haeckel am besten weiss, kein Gewicht zu legen. Man sucht oft an lebenden Spongien vergebens nach ihnen. Was die von Wriht beschriebene Wyvillthomsonia betrifft, welcher Haeckel ebenfalls den Charakter eines Haliphysema retten möchte, so ist dieselbe, wie ich entschieden behaupte, eine junge Stelletta, höchst wahrscheinlich die weit verbreitete, juingst von mir auch bei Neapel nachgewiesene Tisiphonia agariciformis $\mathrm{W}$. T., und gerade durch sie muss der letzte Zweifel an der Schwammnatur von Haliphysema echinoides weichen, welches einstweilen Stelletta echinoides zu nennen wäre.

Damit ist jedoch gegen die Selbstständigkeit der ubrigen Haeckel'schen Gasträaden nichts gesagt. Nur das Eine habe ich 
wollen feststellen, dass, wenn in ihrer Entwicklung jene in Haeckels Arbeit abgebildete Archigastrula vorkommt, sie gerade in diesem fundamentalen Punkte von den Spongien sich entfernen wlirden. Ich habe nicht nur nichts gegen die Gasträatheorie, sondern halte sie fur eine der bedeutendsten Förderungen, welehe unsere Wissenschaft neuerlich erfahren, zumal wenn ihr Urbeber „ihr zunächst mehr eine heuristische als eine causale Bedeutung sichern" will; es ist aber eine sonderbare Ironie des Schicksals, dass sie gerade bei der Thierklasse, von wo aus sie begrindet und einheitlich gestaltet wurde, bei näherem Zusehen nicht ausreicht und nicht zutrifft.

Ich habe noch einige Bemerkungen uber das Verhältniss der larvalen Keimblätter und Zellenmassen zu den Körperschichten der erwachsenen Spongien zu machen. Metschnikoff, auf eigene Beobachtungen gestitzt und die friheren Beobachtungen Schulze's bestätigend, hat die Spongien ganz allgemein für dreiblätterige Thiere erklärt. Diese Aufstellung wird durch Barrois Zustimmung der Schulze'schen Entdeckungen an Kalkschwämmen und Schulze's neue schöne Untersuchungen an Halisarea bekräftigt. Auch ich habe mich an der lebenden Halisarca lobularis von der Richtigkeit von Schul\% e's Beobachtungen tiberzeugt. Somit lässt sich Keller's Einwand, dass bei den Kalkschwämmen die Zellencontouren Kunstprolucte seien, wenigstens nicht verallgemeinern. Am lebenden Sycon raphanus und anderen Syconen sowie an Leuconen ist es mir niemals gelungen, mich von der Zellennatur der Oberflächenschicht zu vergewissern. Die kernartigen Körper, Körnchen und Körnchenhaufen, welche die Kerne der Plattenzellen sein wüden, sind von der verschiedenartigsten Form, Grösse und Zahl, so dass nach dieser Richtung wenigstens Schulze's Bild den Eindruck des Schematischen auf mich machen wüde, wenn ich nicht an seinem eigenen Präparat mich von der Uebereinstimmung tiberzeugt hätte. Bei Ascetta blanca, die bei Neapel sehr gemein ist (Lucia), Asc. primordialis und clathrus, habe ich mich vergeblich abgeplagt, an frischen sowohl wie an vorschriftsmässig zugerichteten Präparaten, das Plattenepithel zu finden. Man muss aber, wie gesagt, zugeben, dass unzweifelhaft wenigstens bei Halisarca das Plattenepithel vorhanden ist, dass es auch bei anderen Spongien vorhanden sein kann und wahrscheinlich vorhanden ist. 
Nun ist es für Schulze, Barrois und Metschnikoff eine ausgemachte Sache, dass diese äussere Plattenzellenschicht der ganzen Cylinderzellenschicht der Larve entspricht, oder mit anderen Worten, dass letztere in ihrer Totalität sich in jene umwandelt. Das halte ich fur unrichtig. Wtirde es sich bestätigen, wie es allen Anschein hat und auch von Schulze als nicht unwahrscheinlich angenommen wird, dass auch nur einige Schwämme, z. B. die Ascetten, dieses Plattenepithel im erwachsenen Zustande nicht besitzen, so ständen die Thatsachen - hier der Uebergang der Geisselzellen in das Plattenepithel, darunter die aus dem primären Entoderm stammende hyaline Schicht, dort kein Plattenepithel, aber eine dem Larvenepiderm entsprechende hyaline Syncytiumschicht, in unversöhnlichem Widerspruch zu einander und zu der Auffassung der Vertheidiger der allgemeinen Dreischichtigkeit. Dieser Widerspruch scheint auch Barrois dunkel vorgeschwebt zu haben, wie ich beim Vergleich seiner Abbildung des jungen Schwammes (Fig. 15) mit den dazu gehörigen und daran anknupfenden Worten entnehme. Die Rolle, welche er die oberste, oft, aber nicht immer sehr regelmässig kranzförmige Abtheilung der Körnerzellen spielen lassen möchte, um jenes hyaline „Mesoderm" des älteren Schwammes daraus herzuleiten, während die hyaline Schicht des jungen Schwammes zum Plattenepithel einschrumpfen misste, ist eine reine Conjectur.

Die vorhandenen Schwierigkeiten bebeben sich, wenn die von keinem jener Forscher, wohl aber von Haeckel aufgeworfene Frage durch die Beobachtung bejaht wtirde, ob denn nicht bei den Schwämmen, welche im erwachsenen Zustande ein Geissel-Plattenepithel als Epidermialschicht besitzen, dasselbe erst nachträglich entstanden sei. Mir scheinen alle bisherigen Beobachtungen uber den Uebergang der Flimmerlarve in den sesshaften Zustand auf das Syncytium als die umgewandelte Cylinderzellensehicht der Larve zu weisen. Warum sollen nicht bei einem Theil der Spongien an der Oberfläche dieser umgeformten Schicht, mit oder ohne Benutzung der vorhandenen, ubrig gebliebenen Kerne Pflasterzellen sich bilden, während bei einem anderen Theile diese Neubildung unterbleibt? Betrachtet man die Grösse der larvalen Cylinderzellen und die Inhaltsmasse der von ihnen gebildeten Schichte und auf der anderen Seite das selbst in seiner Gesammtheit fast verschwindende Plattenepithel 
Oscar Schmidt: Das Larvenstadium von Ascetta primordialis etc. 263

der jungen Spongien, so ist der Unterschied der Quantität so gross, dass schon deshalb an eine Homologie des Inhaltes kaum zu denken. Nach dieser Richtung hin werden wir uns also erst Gewissheit verschaffen mulssen, ehe das definitive Urtheil gefällt werden kann. Mir scheint, dass nur auf diesem Wege die Lösung sich ergeben wird.

\section{Erklärung der Abbildungen anf Tafel XV und XVI.}

1 bis 8. Ascetta primordialis. Haeckel.

1. Stück vom Hinterende der Larve. Bildung einer Wanderzelle aus einer Geisselzelle.

2. Es sind drei Wanderzellen entstanden. Man sieht in das Innere der aufgebrochenen einschichtigen Larve.

3. Häufig vorkommendes Aussehen des Hinterendes bei Druck. Theilweises Heraustreten reifer Wanderzellen.

4. Einschlïpfen der ersten Wander- oder Entodermzelle.

5. Auseinandergebrochene Larve mit sechs Entodermzellen.

6. Hälfte einer Larve von inmen mit zerstreuten Entodermzellen.

7. Larve kurz nach dem Festsetzen.

8. Stück von einer Larve kurz nach dem Festsetzen.

9 bis 14. Ascetta clathrus. Haeckel.

9. Larve am Hinterende geplatzt. Entodermzellen sind herausgetreten.

10. Hinterende der Larve mit hervortretenden Wanderzellen.

11. Ein Stück der aus den Cylinderzellen gebildeten Larvenwand. Daran eine Entodermzelle.

12. Eine Cylinderzelle, zur Errläuterung der Vertheilung der grünen Pigmentkörnchen.

13. Zwei Cylinderzellen mit zufällig an ihnen haftenden zwei Entodermzellen.

14. Frische amöboide Entodermzellen. 


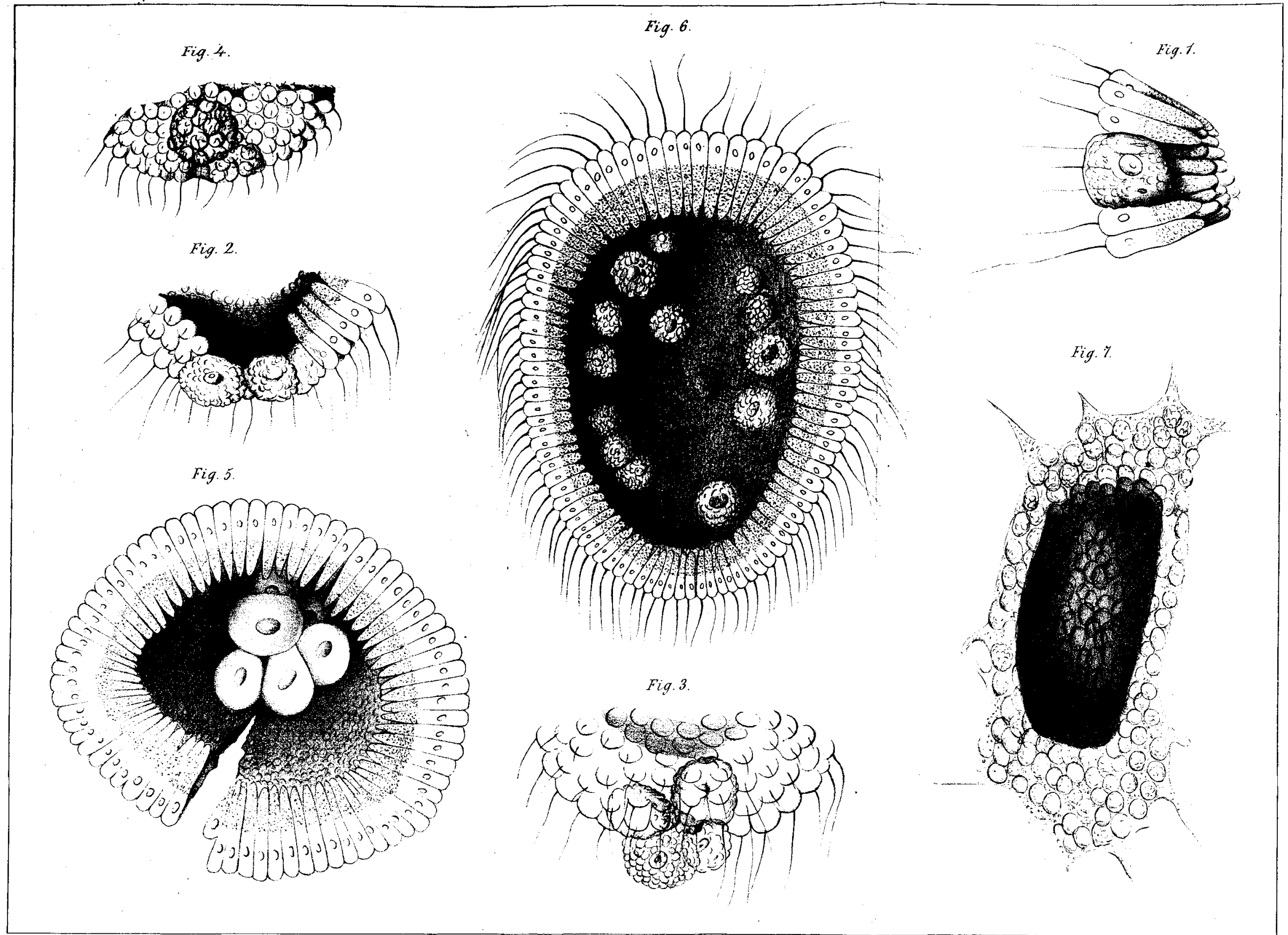


Archio f mikroshop Anatomie Bd. Xll.

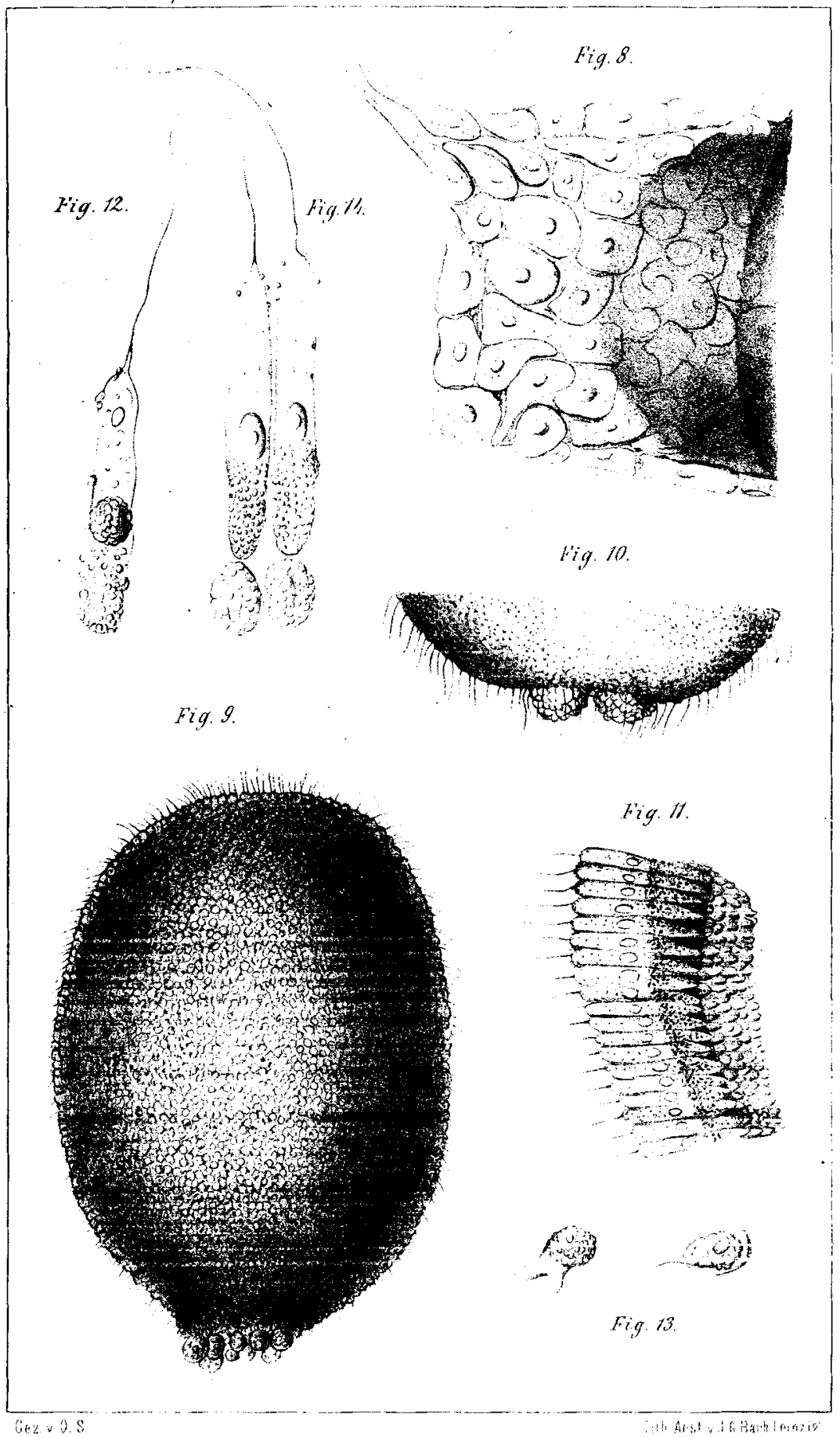

